Network Working Group

Request for Comments: 2575

Obsoletes: 2275

Category: Standards Track
B. Wijnen

IBM T. J. Watson Research

R. Presuhn

BMC Software, Inc.

K. McCloghrie

Cisco Systems, Inc.

April 1999

\title{
View-based Access Control Model (VACM) for the Simple Network Management Protocol (SNMP)
}

Status of this Memo

This document specifies an Internet standards track protocol for the Internet community, and requests discussion and suggestions for improvements. Please refer to the current edition of the "Internet Official Protocol standards" (STD 1) for the standardization state and status of this protocol. Distribution of this memo is unlimited.

Copyright Notice

Copyright (C) The Internet Society (1999). All Rights Reserved.

Abstract

This document describes the View-based Access Control Model for use in the SNMP architecture [RFC2571]. It defines the Elements of Procedure for controlling access to management information. This document also includes a MIB for remotely managing the configuration parameters for the View-based Access Control Model.

Table of Contents

1. Introduction

1.2. Access Control

1.3. Local Configuration Datastore

2. Elements of the Model 3

2.1. Groups 3

2.2. securityLevel 4

2.3. Contexts 4

2.4. MIB Views and View Families 4

2.4.1. View Subtree 5

2.4.2. ViewTreeFamily 25

2.5. Access Policy 6

3. Elements of Procedure 6

3.1. Overview of isAccessAllowed Process 8

3.2. Processing the isAccessAllowed Service Request 9 
4. Definitions 10

5. Intellectual Property 27

6. Acknowledgements 28

7. Security Considerations 29

7.1. Recommended Practices 29

7.2. Defining Groups 30

7.3. Conformance 30

7.4. Access to the SNMP-VIEW-BASED-ACM-MIB 30

8. References 31

9. Editors' Addresses 32

A.1. Installation Parameters 33

B. Change Log $\quad 37$

C. Full Copyright Statement 38

1. Introduction

The Architecture for describing Internet Management Frameworks [RFC2571] describes that an SNMP engine is composed of:

1) a Dispatcher

2) a Message Processing Subsystem,

3) a Security Subsystem, and

4) an Access Control Subsystem.

Applications make use of the services of these subsystems.

It is important to understand the SNMP architecture and its terminology to understand where the View-based Access Control Model described in this document fits into the architecture and interacts with other subsystems within the architecture. The reader is expected to have read and understood the description and terminology of the SNMP architecture, as defined in [RFC2571].

The Access Control Subsystem of an SNMP engine has the responsibility for checking whether a specific type of access (read, write, notify) to a particular object (instance) is allowed.

It is the purpose of this document to define a specific model of the Access Control Subsystem, designated the View-based Access Control Model. Note that this is not necessarily the only Access Control Model.

The key words "MUST", "MUST NOT", "REQUIRED", "SHALL", "SHALL NOT", "SHOULD", "SHOULD NOT", "RECOMMENDED", "MAY", and "OPTIONAL" in this document are to be interpreted as described in [RFC2119]. 


\subsection{Access Control}

Access Control occurs (either implicitly or explicitly) in an SNMP entity when processing SNMP retrieval or modification request messages from an SNMP entity. For example a Command Responder application applies Access Control when processing requests that it received from a Command Generator application. These requests contain Read Class and Write Class PDUs as defined in [RFC2571].

Access Control also occurs in an SNMP entity when an SNMP notification message is generated (by a Notification Originator application). These notification messages contain Notification Class PDUs as defined in [RFC2571].

The View-based Access Control Model defines a set of services that an application (such as a Command Responder or a Notification Originator application) can use for checking access rights. It is the responsibility of the application to make the proper service calls for access checking.

\subsection{Local Configuration Datastore}

To implement the model described in this document, an SNMP entity needs to retain information about access rights and policies. This information is part of the SNMP engine's Local Configuration Datastore (LCD). See [RFC2571] for the definition of LCD.

In order to allow an SNMP entity's LCD to be remotely configured, portions of the LCD need to be accessible as managed objects. A MIB module, the View-based Access Control Model Configuration MIB, which defines these managed object types is included in this document.

2. Elements of the Model

This section contains definitions to realize the access control service provided by the View-based Access Control Model.

\subsection{Groups}

A group is a set of zero or more <securityModel, securityName> tuples on whose behalf SNMP management objects can be accessed. A group defines the access rights afforded to all securityNames which belong to that group. The combination of a securityModel and a securityName maps to at most one group. A group is identified by a groupName.

The Access Control module assumes that the securityName has already been authenticated as needed and provides no further authentication of its own. 
The View-based Access Control Model uses the securityModel and the securityName as inputs to the Access Control module when called to check for access rights. It determines the groupName as a function of securityModel and securityName.

\section{2. securityLevel}

Different access rights for members of a group can be defined for different levels of security, i.e., noAuthNoPriv, authNoPriv, and authPriv. The securitylevel identifies the level of security that will be assumed when checking for access rights. See the SNMP Architecture document [RFC2571] for a definition of securityLevel.

The View-based Access Control Model requires that the securitylevel is passed as input to the Access Control module when called to check for access rights.

\subsection{Contexts}

An SNMP context is a collection of management information accessible by an SNMP entity. An item of management information may exist in more than one context. An SNMP entity potentially has access to many contexts. Details about the naming of management information can be found in the SNMP Architecture document [RFC2571].

The View-based Access Control Model defines a vacmContextTable that lists the locally available contexts by contextName.

\subsection{MIB Views and View Families}

For security reasons, it is often valuable to be able to restrict the access rights of some groups to only a subset of the management information in the management domain. To provide this capability, access to a context is via a "MIB view" which details a specific set of managed object types (and optionally, the specific instances of object types) within that context. For example, for a given context, there will typically always be one MIB view which provides access to all management information in that context, and often there will be other MIB views each of which contains some subset of the information. So, the access allowed for a group can be restricted in the desired manner by specifying its rights in terms of the particular (subset) MIB view it can access within each appropriate context.

Since managed object types (and their instances) are identified via the tree-like naming structure of ISO's OBJECT IDENTIFIERs [ISOASN.1, RFC2578], it is convenient to define a MIB view as the combination of a set of "view subtrees", where each view subtree is a 
subtree within the managed object naming tree. Thus, a simple MIB view (e.g., all managed objects within the Internet Network Management Framework) can be defined as a single view subtree, while more complicated MIB views (e.g., all information relevant to a particular network interface) can be represented by the union of multiple view subtrees.

While any set of managed objects can be described by the union of some number of view subtrees, situations can arise that would require a very large number of view subtrees. This could happen, for example, when specifying all columns in one conceptual row of a MIB table because they would appear in separate subtrees, one per column, each with a very similar format. Because the formats are similar, the required set of subtrees can easily be aggregated into one structure. This structure is named a family of view subtrees after the set of subtrees that it conceptually represents. A family of view subtrees can either be included or excluded from a MIB view.

\subsubsection{View Subtree}

A view subtree is the set of all MIB object instances which have a common ASN.1 OBJECT IDENTIFIER prefix to their names. A view subtree is identified by the OBJECT IDENTIFIER value which is the longest OBJECT IDENTIFIER prefix common to all (potential) MIB object instances in that subtree.

\subsubsection{ViewTreeFamily}

A family of view subtrees is a pairing of an OBJECT IDENTIFIER value (called the family name) together with a bit string value (called the family mask). The family mask indicates which sub-identifiers of the associated family name are significant to the family's definition.

For each possible managed object instance, that instance belongs to a particular ViewTreeFamily if both of the following conditions are true:

- the OBJECT IDENTIFIER name of the managed object instance contains at least as many sub-identifiers as does the family name, and

- each sub-identifier in the OBJECT IDENTIFIER name of the managed object instance matches the corresponding sub-identifier of the family name whenever the corresponding bit of the associated family mask is non-zero. 
When the configured value of the family mask is all ones, the view subtree family is identical to the single view subtree identified by the family name.

When the configured value of the family mask is shorter than required to perform the above test, its value is implicitly extended with ones. Consequently, a view subtree family having a family mask of zero length always corresponds to a single view subtree.

\subsection{Access Policy}

The View-based Access Control Model determines the access rights of a group, representing zero or more securityNames which have the same access rights. For a particular context, identified by contextName, to which a group, identified by groupName, has access using a particular securitymodel and securitylevel, that group's access rights are given by a read-view, a write-view and a notify-view.

The read-view represents the set of object instances authorized for the group when reading objects. Reading objects occurs when processing a retrieval operation (when handling Read Class PDUs).

The write-view represents the set of object instances authorized for the group when writing objects. Writing objects occurs when processing a write operation (when handling Write Class PDUs).

The notify-view represents the set of object instances authorized for the group when sending objects in a notification, such as when sending a notification (when sending Notification Class PDUs).

\section{Elements of Procedure}

This section describes the procedures followed by an Access Control module that implements the View-based Access Control Model when checking access rights as requested by an application (for example a Command Responder or a Notification Originator application). The abstract service primitive is:

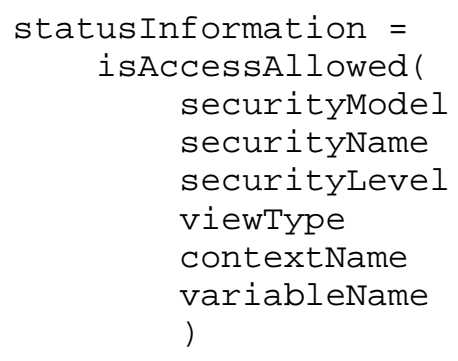

-- success or errorIndication

-- Security Model in use

-- principal who wants access

-- Level of Security

-- read, write, or notify view

-- context containing variableName

-- OID for the managed object 
The abstract data elements are:

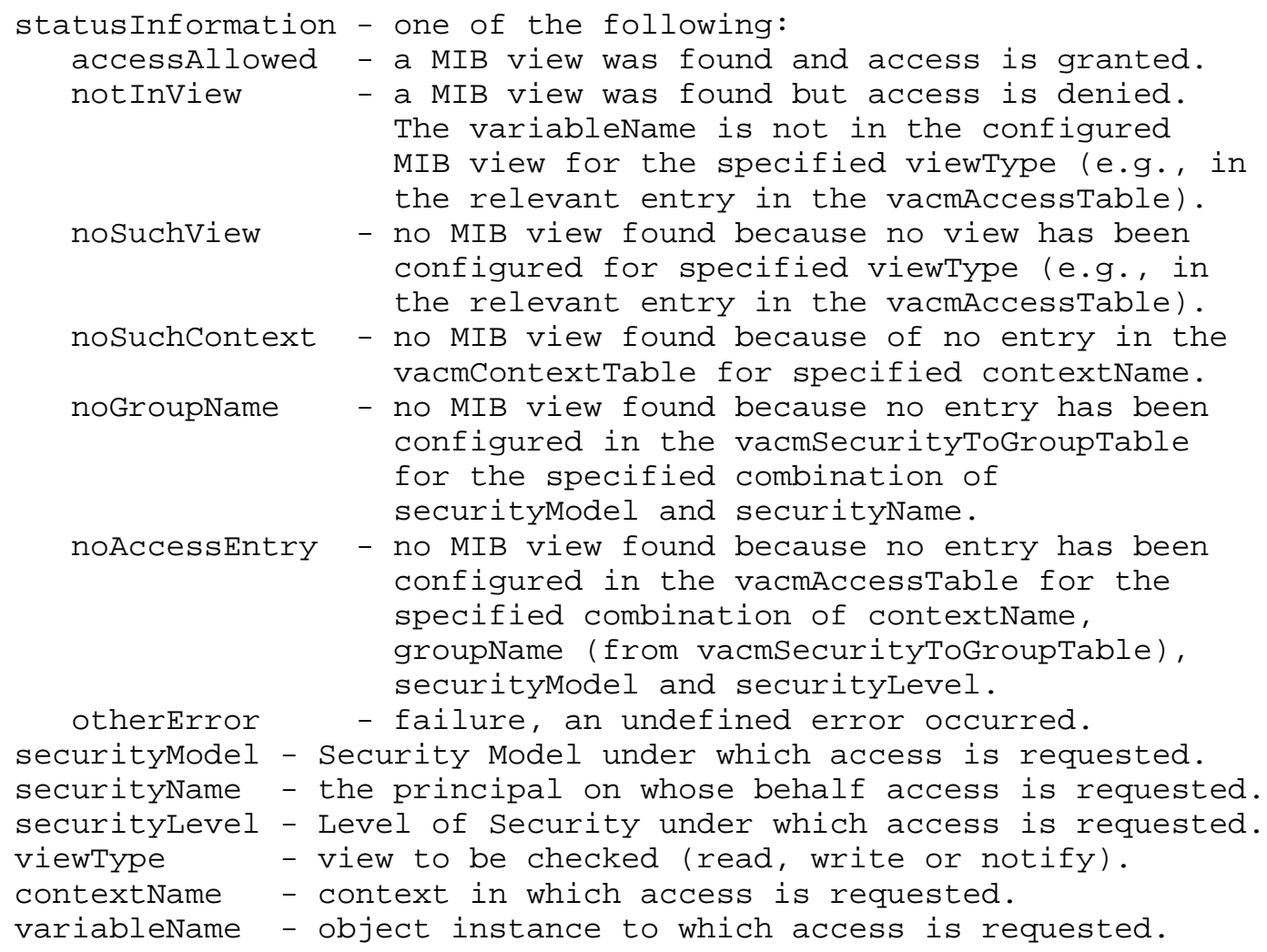




\subsection{Overview of isAccessAllowed Process}

The following picture shows how the decision for access control is made by the View-based Access Control Model.

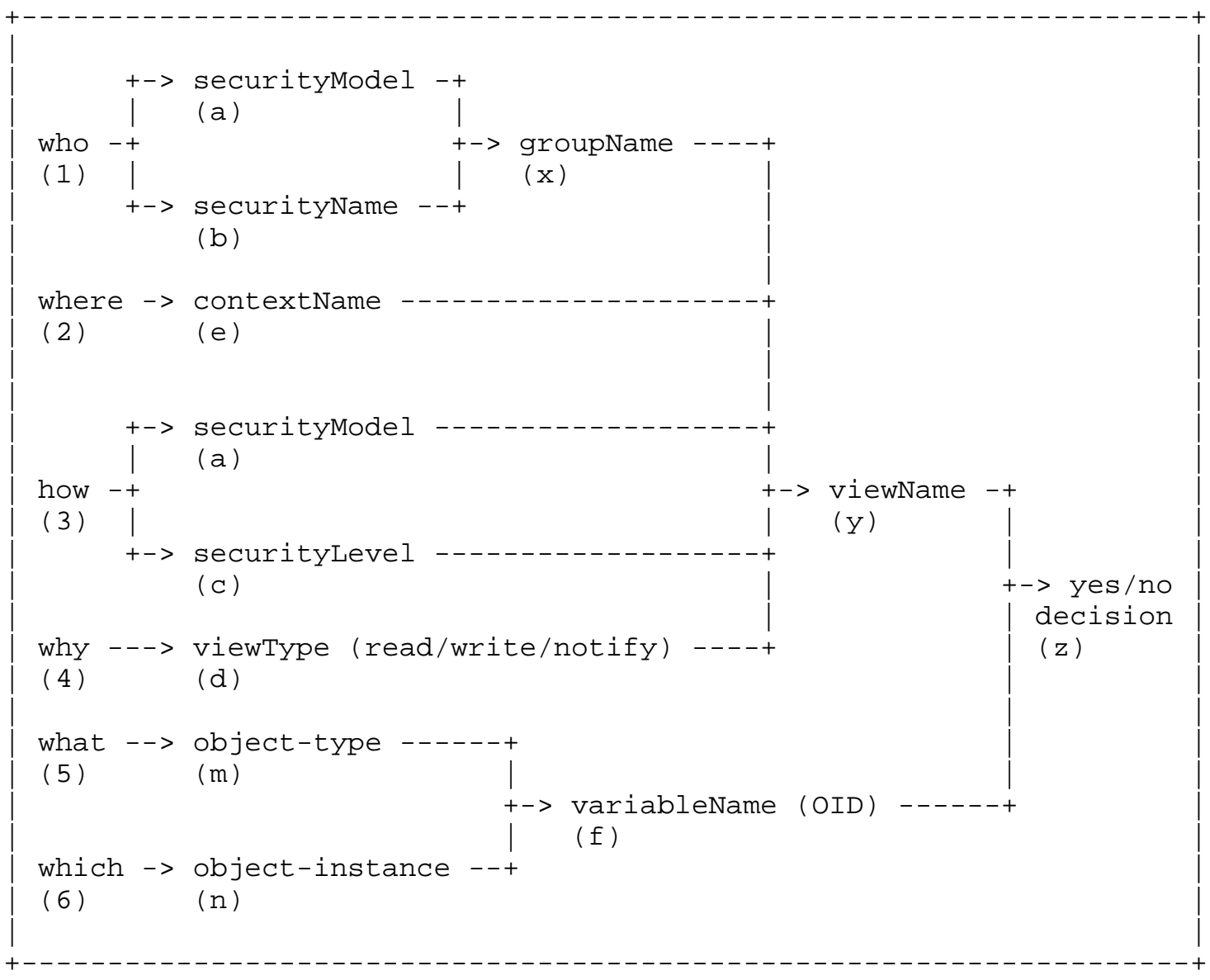

How the decision for isAccessAllowed is made.

1) Inputs to the isAccessAllowed service are:
(a)
(b)
securityModel
-- Security Model in use
(c)
securityName
-- principal who wants to access
(d)
securityLevel
-- Level of Security
viewType
-- read, write, or notify view
(e)
contextName
-- context containing variableName 


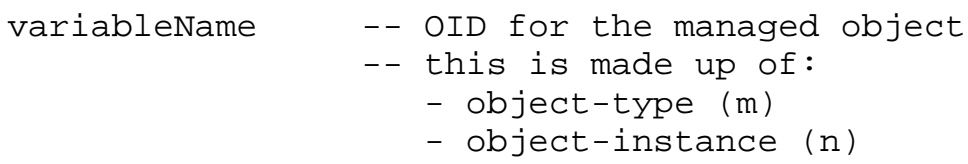

2) The partial "who" (1), represented by the securityModel (a) and the securityName (b), are used as the indices $(a, b)$ into the vacmSecurityToGroupTable to find a single entry that produces a group, represented by groupName (x).

3) The "where" (2), represented by the contextName (e), the "who", represented by the groupName (x) from the previous step, and the "how" (3), represented by securityModel (a) and securitylevel (c), are used as indices $(e, x, a, c)$ into the vacmAccesstable to find a single entry that contains three MIB views.

4) The "why" (4), represented by the viewType (d), is used to select the proper MIB view, represented by a viewName (y), from the vacmAccessentry selected in the previous step. This viewName (y) is an index into the vacmViewTreeFamilyTable and selects the set of entries that define the variableNames which are included in or excluded from the MIB view identified by the viewName (y).

5) The "what" (5) type of management data and "which" (6) particular instance, represented by the variableName (f), is then checked to be in the MIB view or not, e.g., the yes/no decision (z).

\subsection{Processing the isAccessAllowed Service Request}

This section describes the procedure followed by an Access Control module that implements the View-based Access Control Model whenever it receives an isAccessAllowed request.

1) The vacmcontextTable is consulted for information about the SNMP context identified by the contextName. If information about this SNMP context is absent from the table, then an errorIndication (noSuchcontext) is returned to the calling module.

2) The vacmSecurityToGroupTable is consulted for mapping the securityModel and securityName to a groupName. If the information about this combination is absent from the table, then an errorIndication (noGroupName) is returned to the calling module.

3) The vacmAccesstable is consulted for information about the groupName, contextName, securityModel and securitylevel. If information about this combination is absent from the table, then an errorIndication (noAccessentry) is returned to the calling module. 
4) a) If the viewType is "read", then the read view is used for checking access rights.

b) If the viewType is "write", then the write view is used for checking access rights.

c) If the viewType is "notify", then the notify view is used for checking access rights.

If the view to be used is the empty view (zero length viewName) then an errorIndication (noSuchView) is returned to the calling module.

5) a) If there is no view configured for the specified viewType, then an errorIndication (nosuchView) is returned to the calling module.

b) If the specified variableName (object instance) is not in the MIB view (see DESCRIPTION clause for vacmViewTreeFamilyTable in section 4), then an errorIndication (notInView) is returned to the calling module.

Otherwise,

C) The specified variableName is in the MIB view. A statusinformation of success (accessAllowed) is returned to the calling module.

4. Definitions

SNMP-VIEW-BASED-ACM-MIB DEFINITIONS : := BEGIN

IMPORTS

MODULE-COMPLIANCE, OBJECT-GROUP FROM SNMPV2-CONF

MODULE-IDENTITY, OBJECT-TYPE,

snmpModules

TestAndIncr,

RowStatus, StorageType FROM SNMPv2-TC

SnmpAdminstring, SnmpsecurityLevel, SnmpsecurityModel

FROM SNMPV2-SMI

FROM SNMP-FRAMEWORK-MIB;

SnmpVacmMIB MODULE-IDENTITY

LAST-UPDATED "9901200000Z" -- 20 Jan 1999, midnight

ORGANIZATION "SNMPV3 Working Group"

CONTACT-INFO "WG-email: snmpv3Qlists.tislabs.com Subscribe: majordomolists.tislabs.com

In message body: subscribe snmpv3

Wijnen, et al.

Standards Track

[Page 10] 


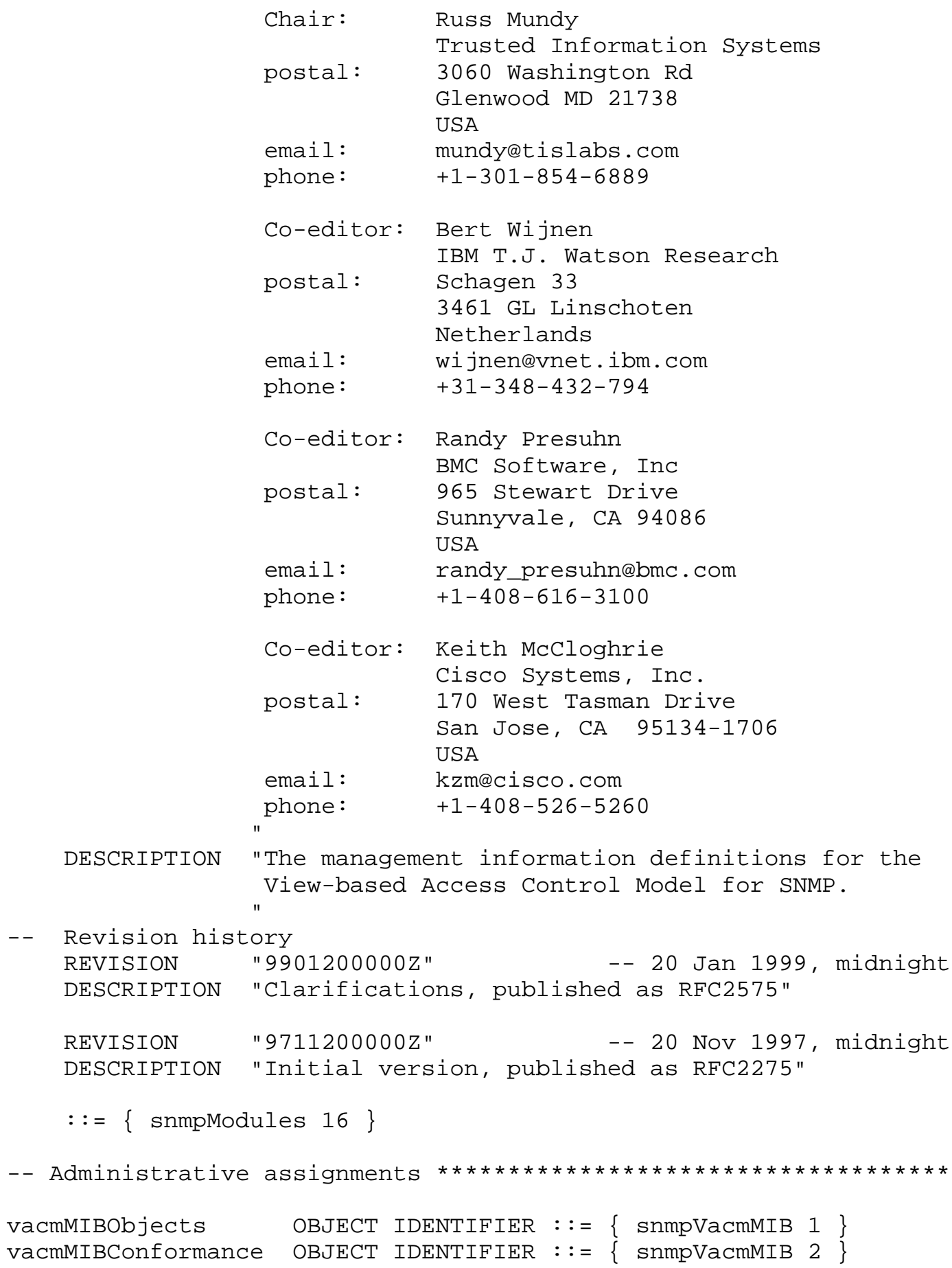




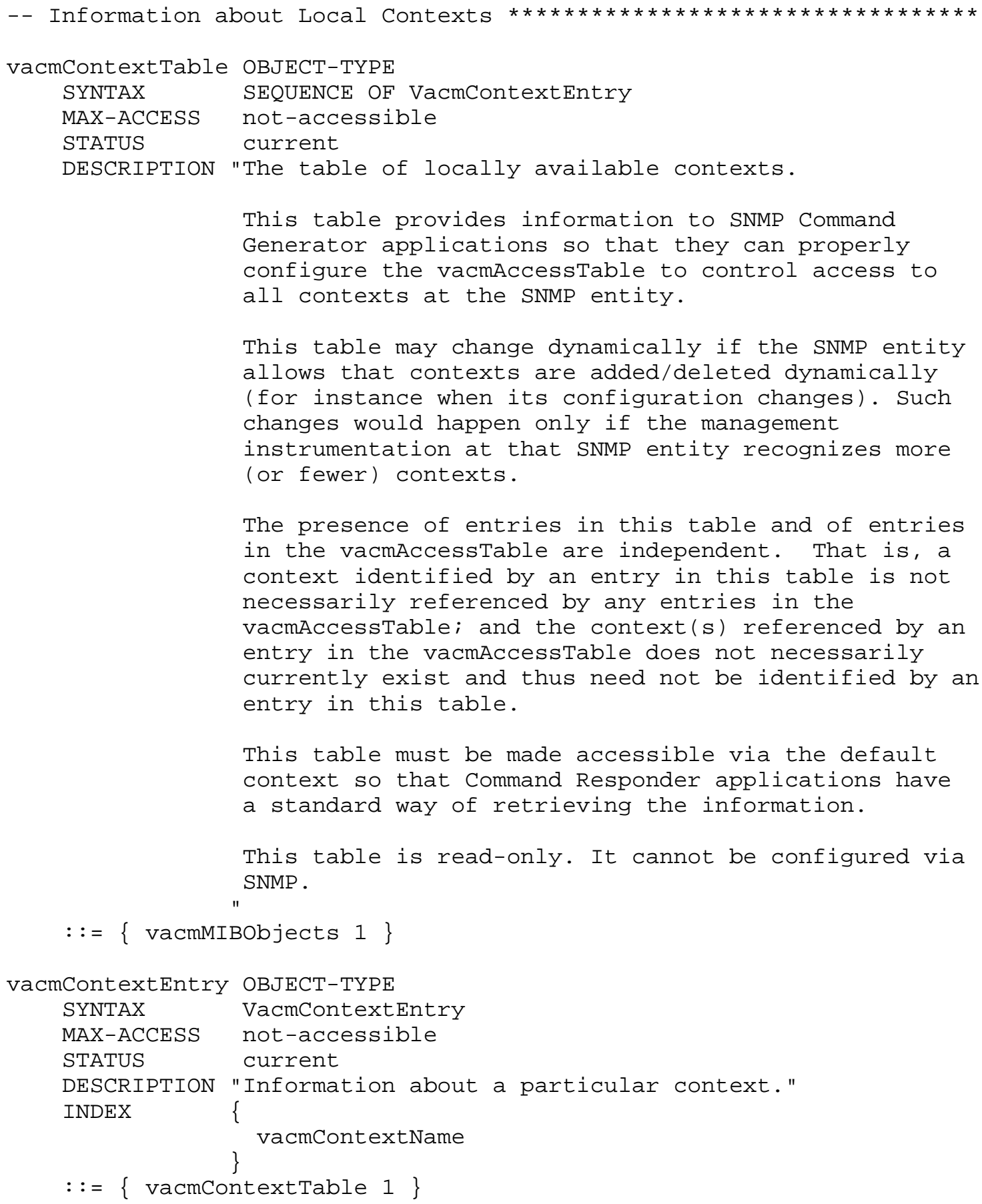




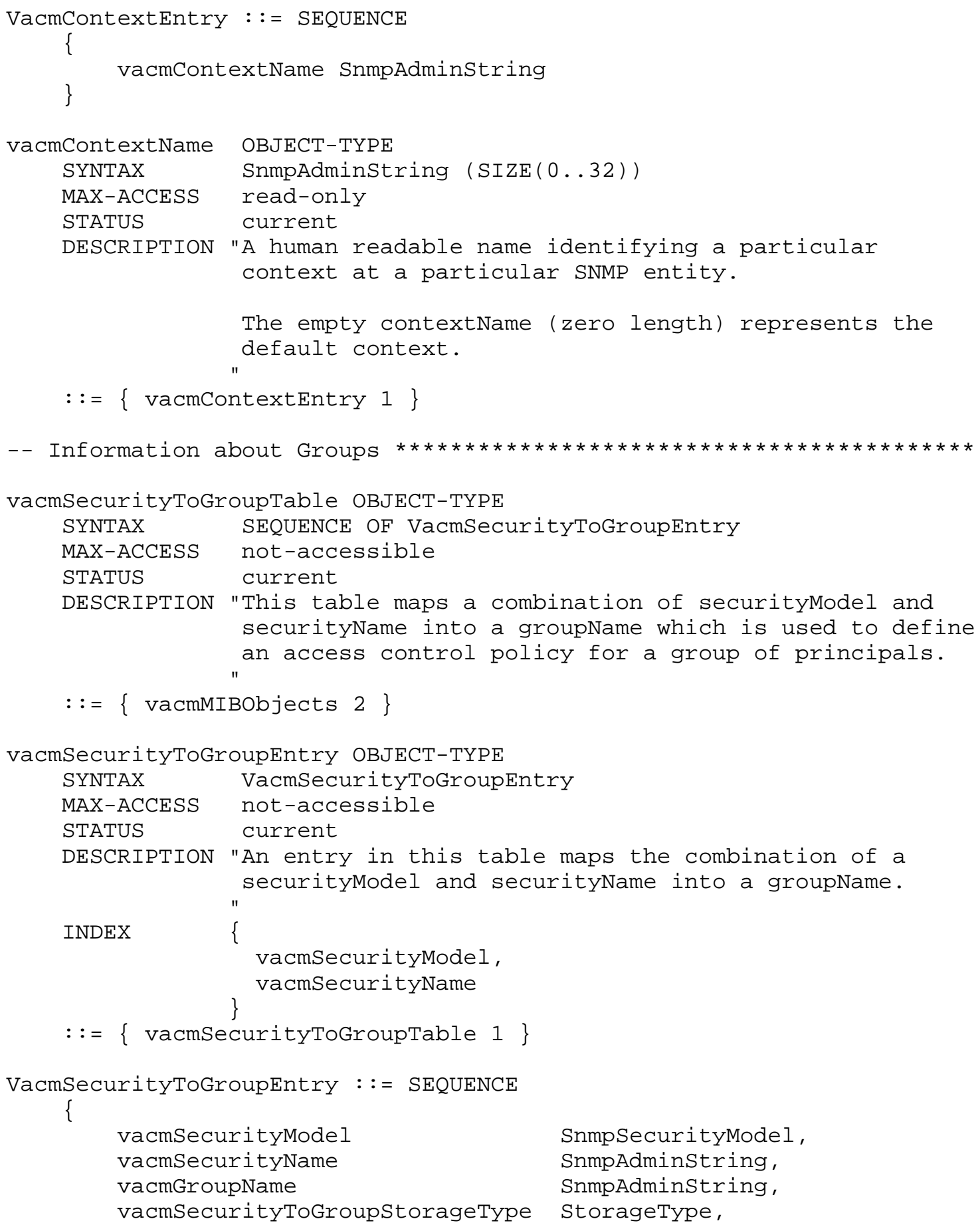


\}

vacmSecurityToGroupstatus Rowstatus

vacmSecurityModel OBJECT-TYPE

SYNTAX SnmpsecurityModel (1..2147483647)

MAX-ACCESS not-accessible

STATUS current

DESCRIPTION "The Security Model, by which the vacmSecurityName referenced by this entry is provided.

Note, this object may not take the 'any' (0) value.

$::=\{$ vacmSecurityToGroupEntry 1$\}$

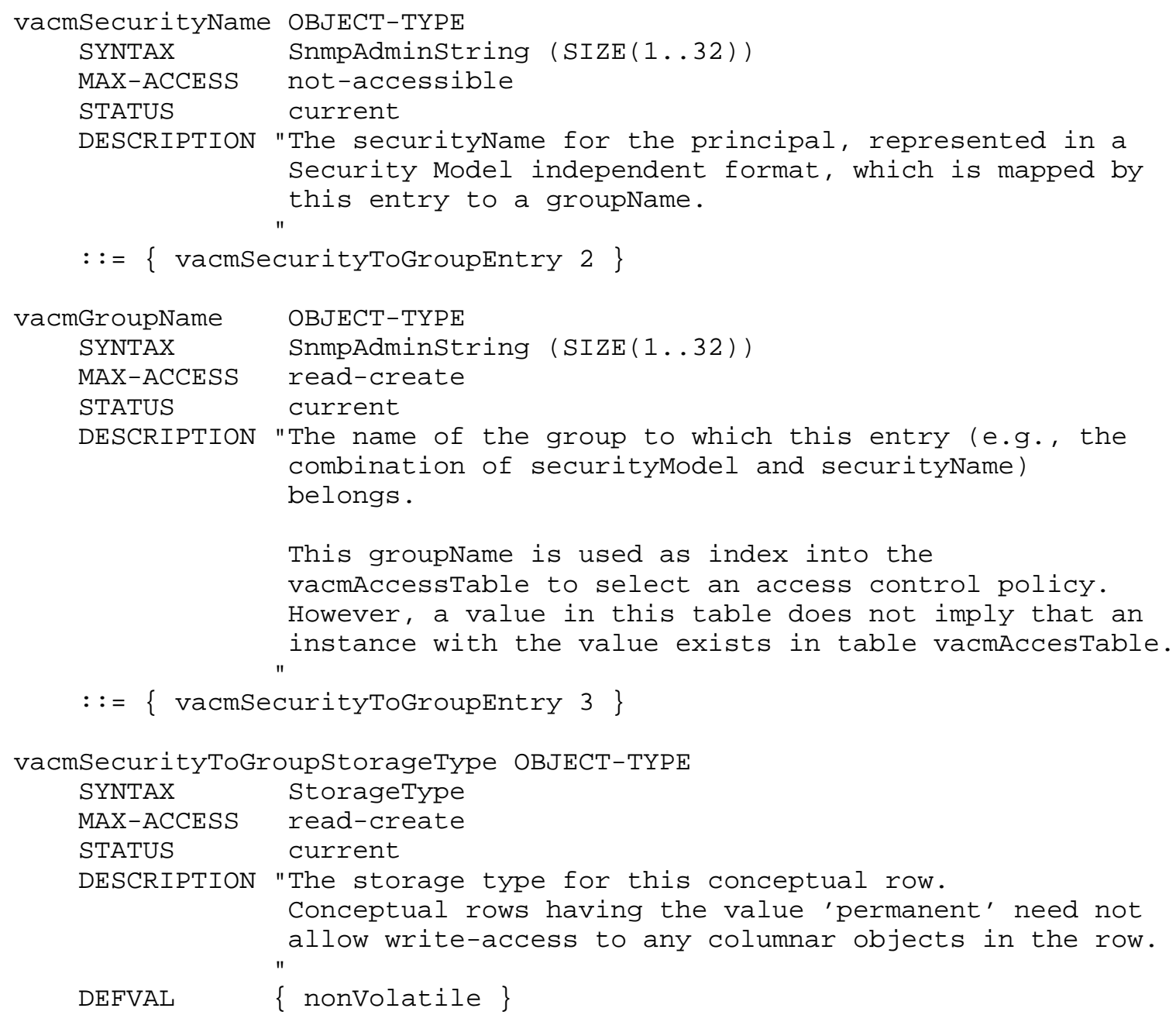


$::=\{$ vacmSecurityToGroupEntry 4$\}$

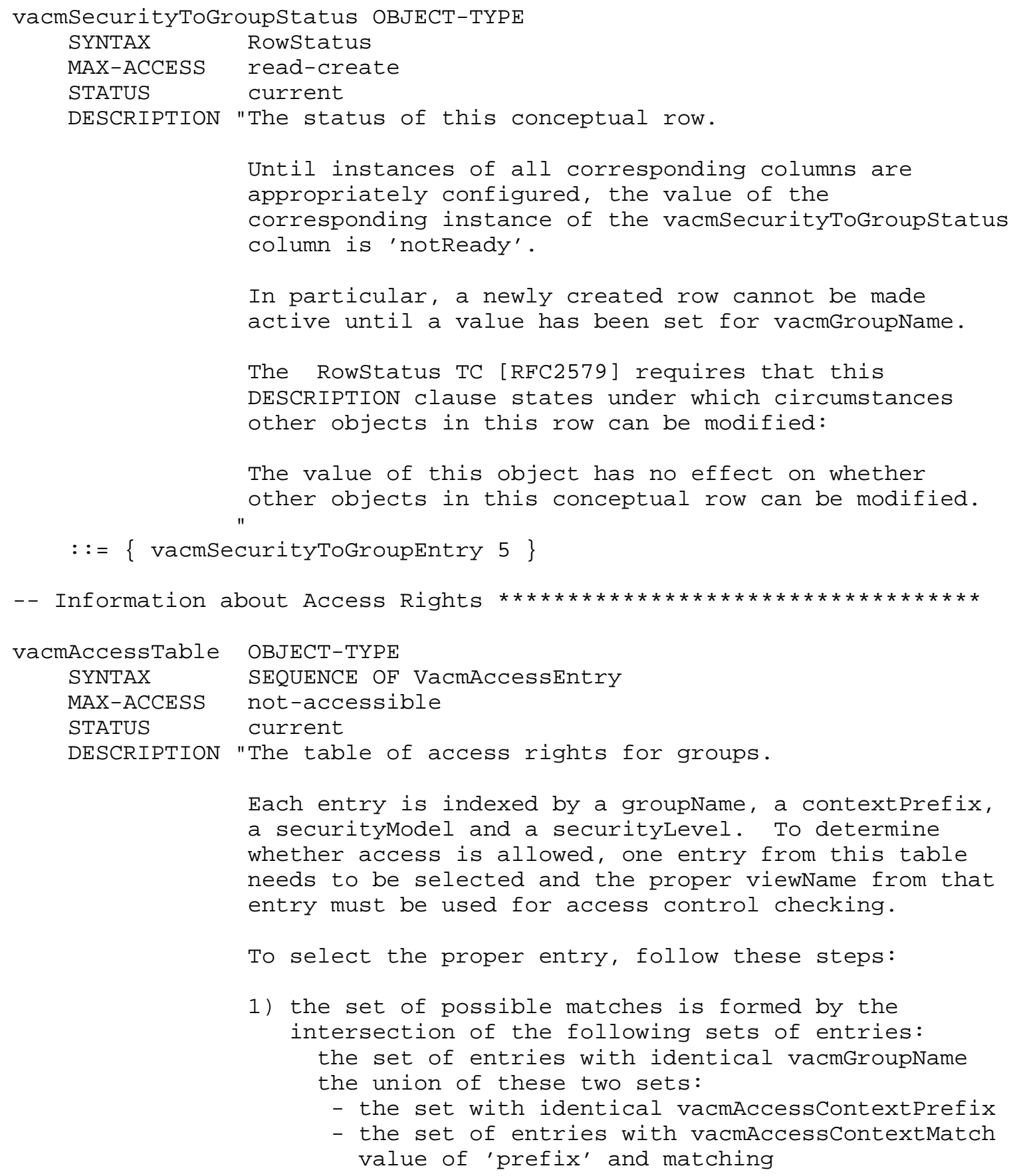




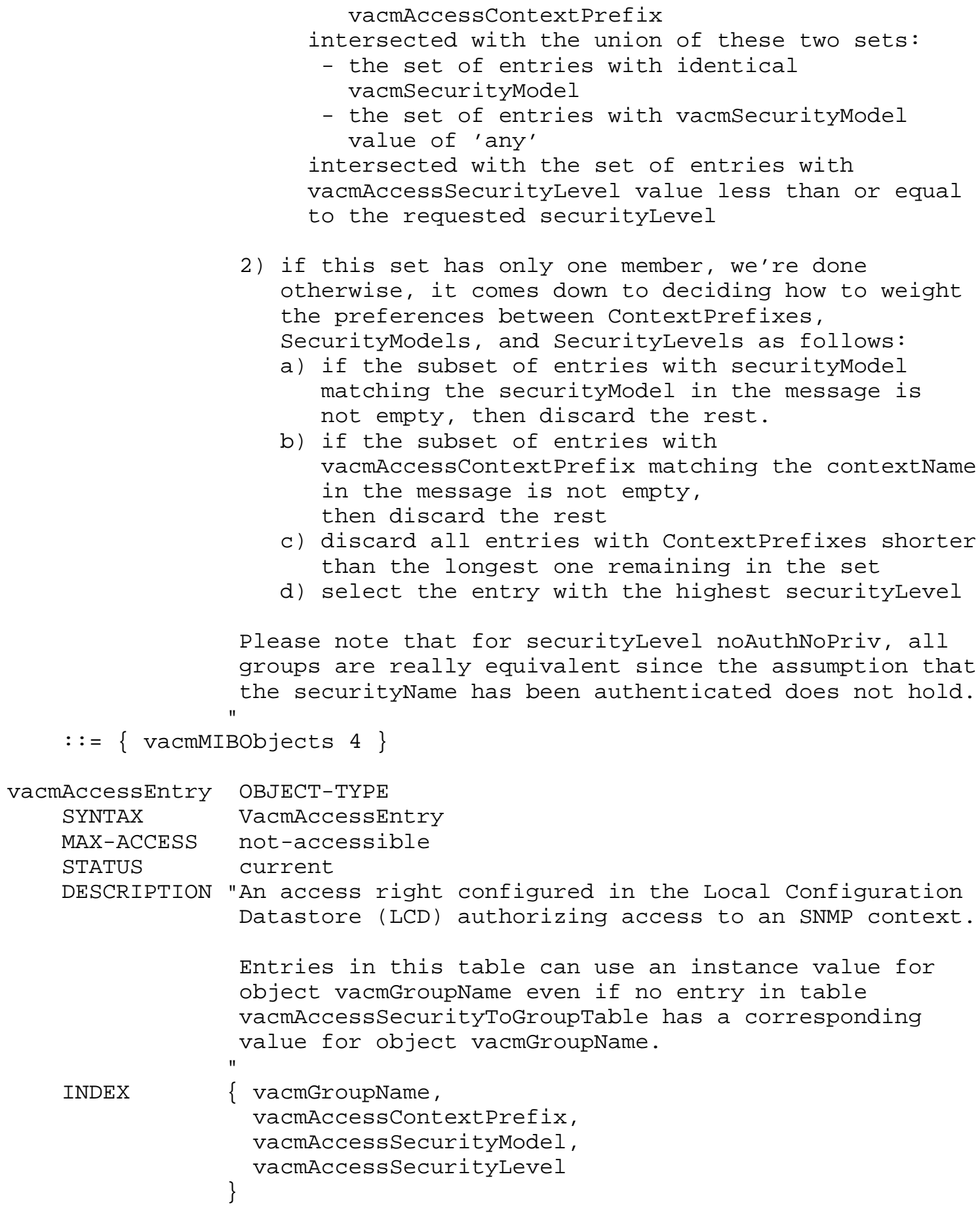


$::=\{$ vacmAccesstable 1$\}$

VacmAccessEntry : := SEQUENCE

\{

vacmAccesscontextPrefix

SnmpAdminstring,

vacmAccess SecurityModel

SnmpsecurityModel, vacmAccess SecurityLevel SnmpsecurityLevel, vacmAccesscontextMatch INTEGER, vacmAccessReadViewName SnmpAdminString, vacmAccessWriteViewName vacmAccessNotifyViewName SnmpAdminstring, vacmAccess StorageType SnmpAdminString, vacmAccessstatus StorageType,

\} Rowstatus

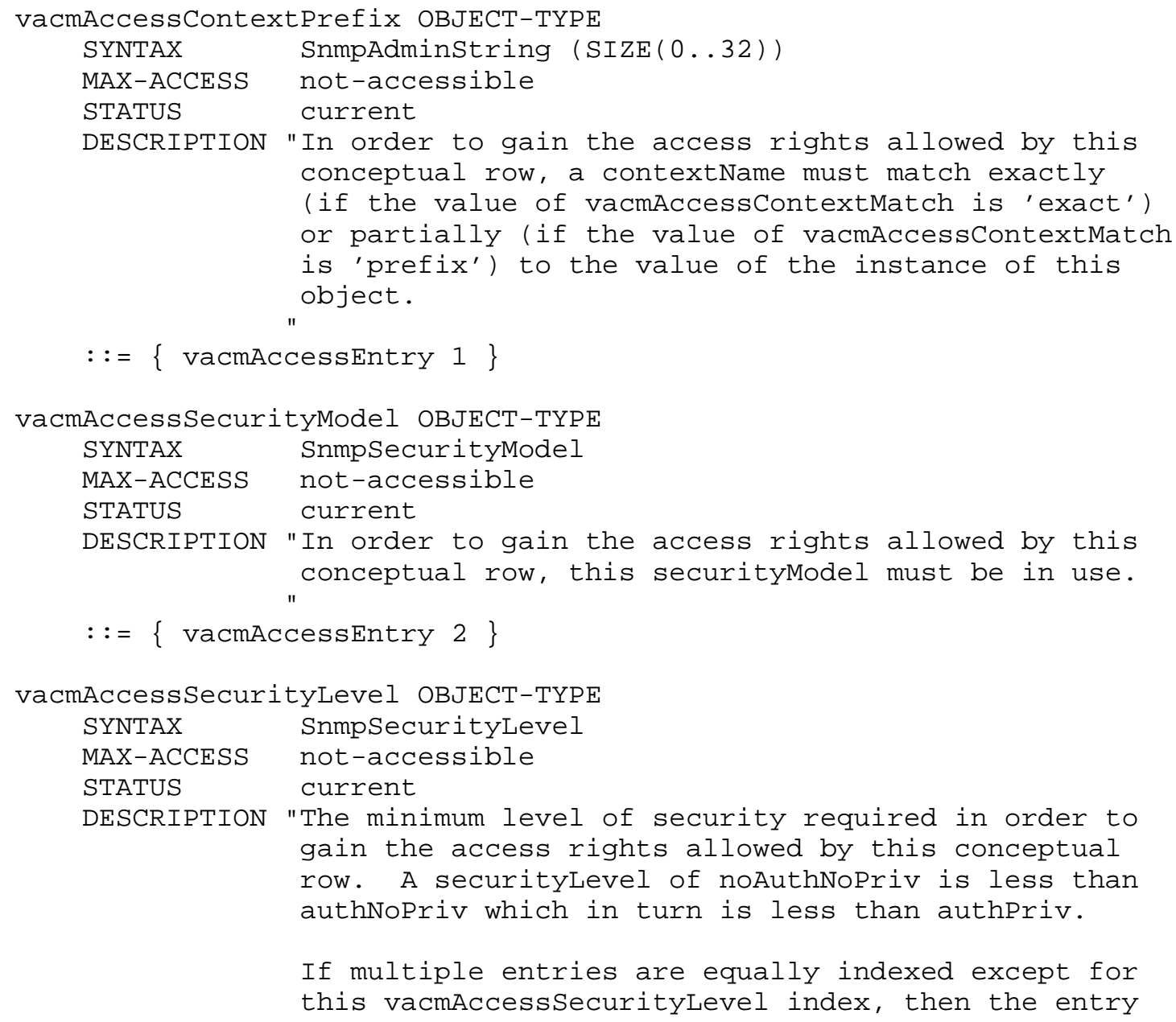




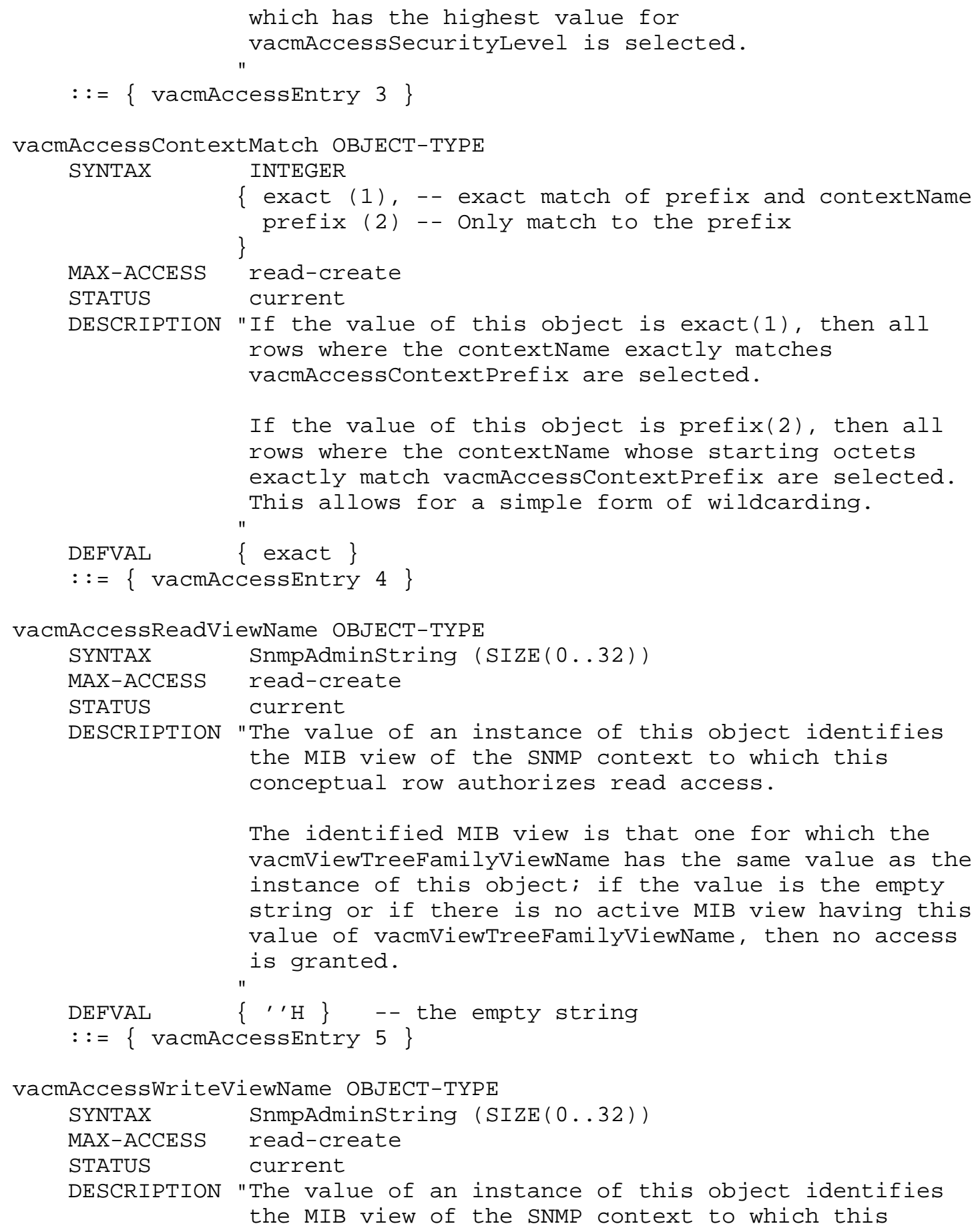




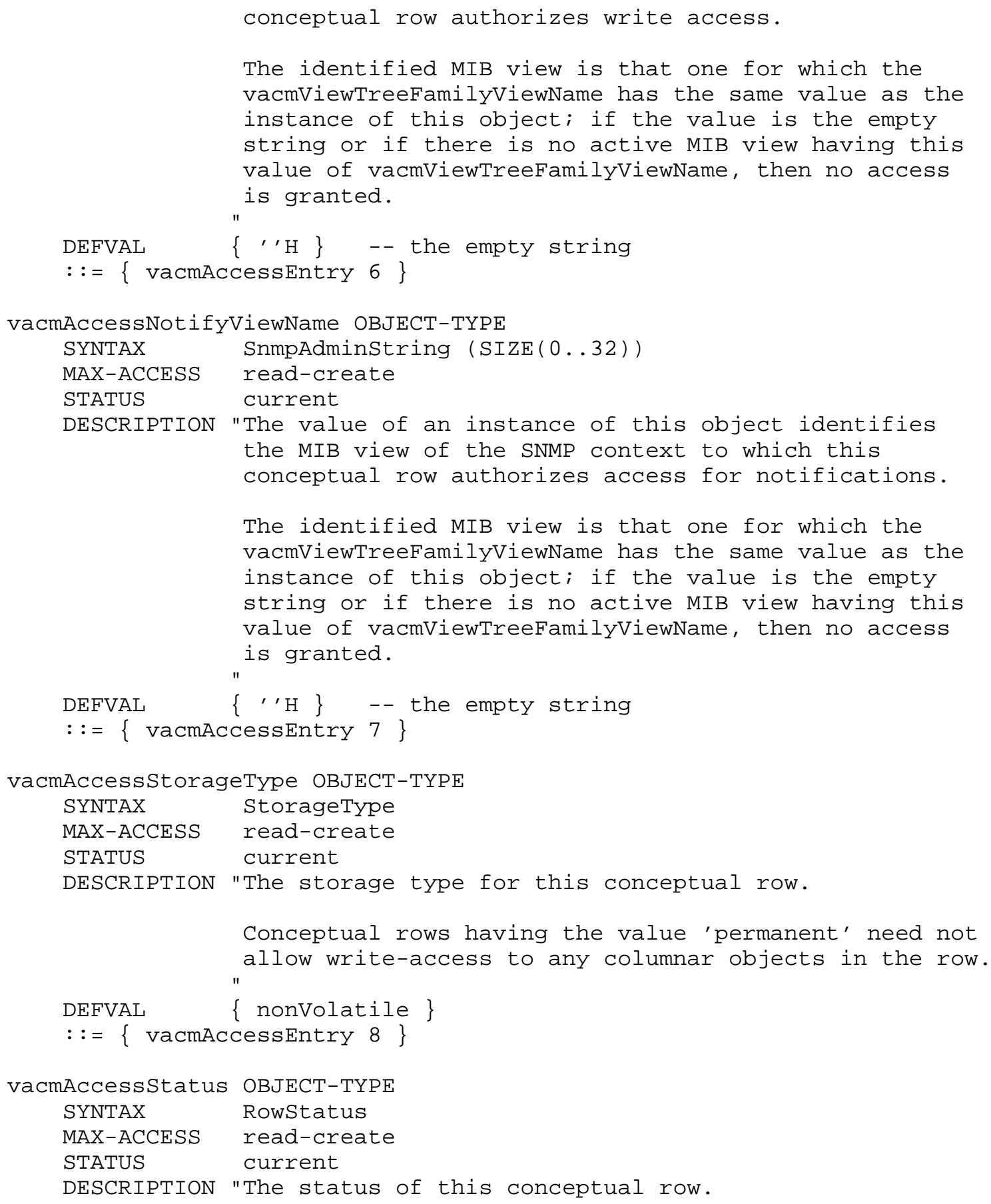


The Rowstatus TC [RFC2579] requires that this DESCRIPTION clause states under which circumstances other objects in this row can be modified:

The value of this object has no effect on whether other objects in this conceptual row can be modified. $::=\{$ vacmAccessEntry 9$\}$

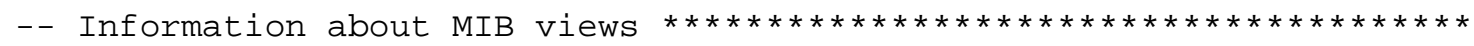

-- Support for instance-level granularity is optional.

\section{$--$}

-- In some implementations, instance-level access control

-- granularity may come at a high performance cost. Managers

-- should avoid requesting such configurations unnecessarily.

vacmMIBViews OBJECT IDENTIFIER : := \{vacmMIBObjects 5$\}$

vacmViewSpinLock OBJECT-TYPE SYNTAX TestAndIncr

MAX-ACCESS read-write

STATUS current

DESCRIPTION "An advisory lock used to allow cooperating SNMP Command Generator applications to coordinate their use of the set operation in creating or modifying views.

When creating a new view or altering an existing view, it is important to understand the potential interactions with other uses of the view. The vacmViewSpinLock should be retrieved. The name of the view to be created should be determined to be unique by the SNMP Command Generator application by consulting the vacmViewTreeFamilyTable. Finally, the named view may be created (Set), including the advisory lock. If another SNMP Command Generator application has altered the views in the meantime, then the spin lock's value will have changed, and so this creation will fail because it will specify the wrong value for the spin lock.

Since this is an advisory lock, the use of this lock is not enforced.

$::=\{$ vacmMIBViews 1$\}$ 


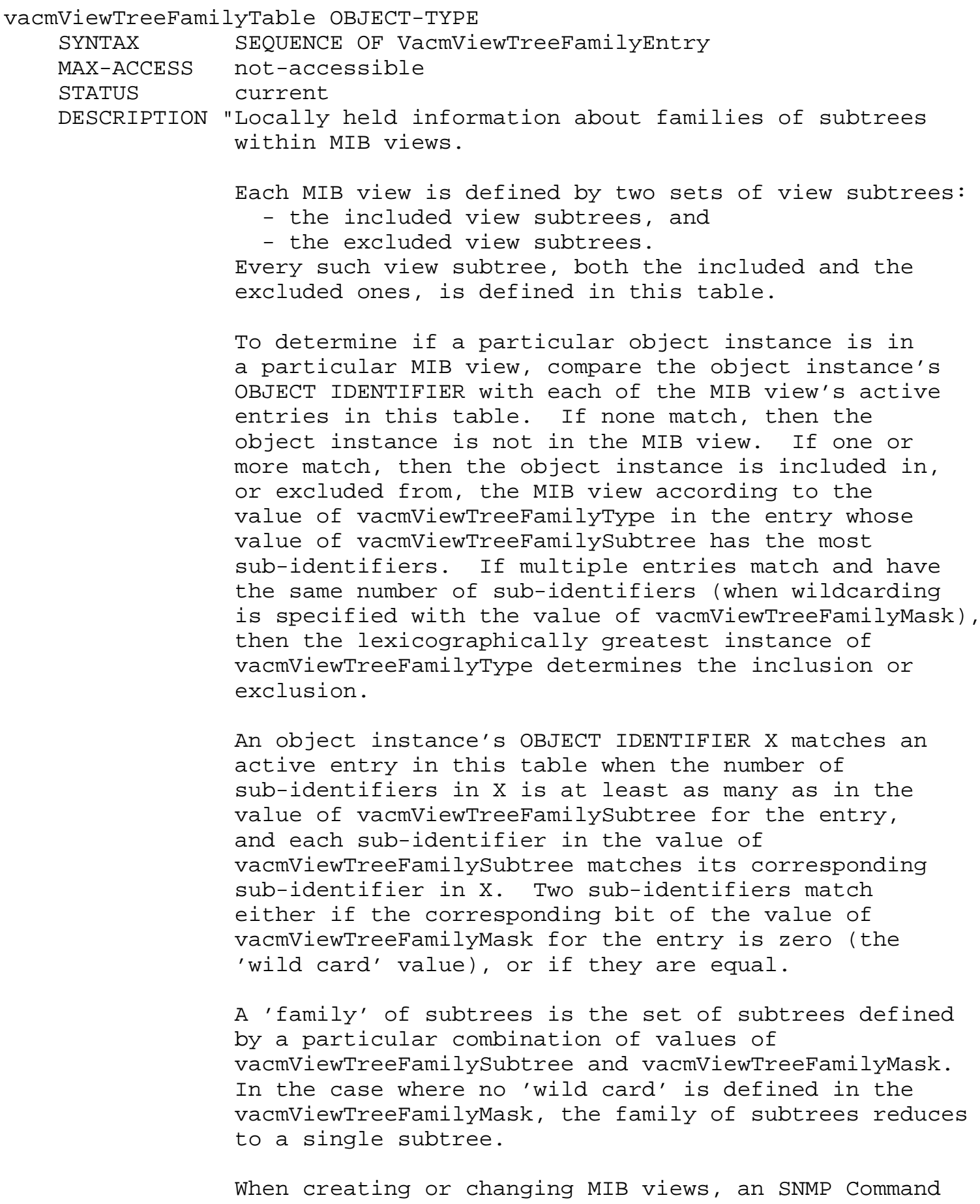




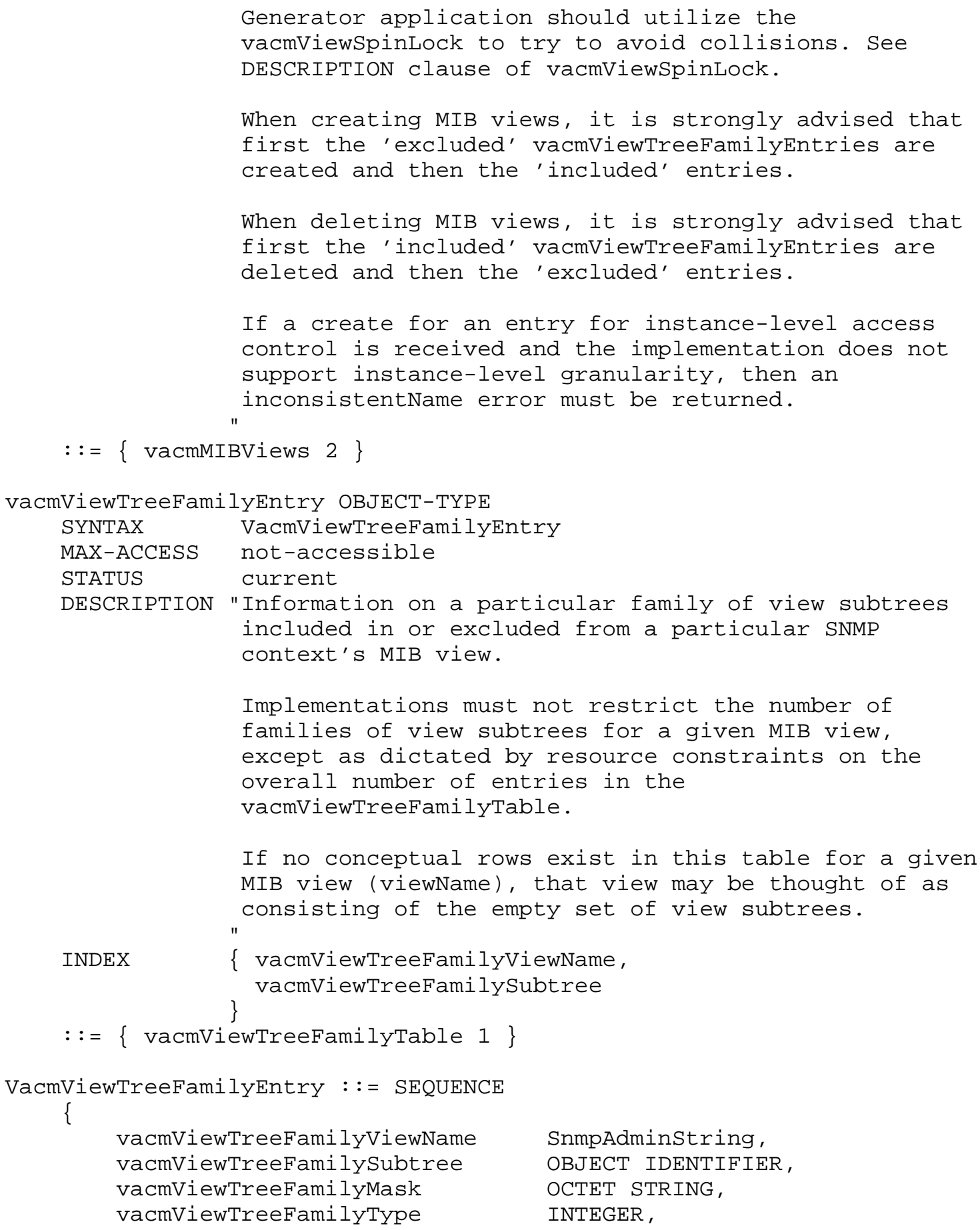




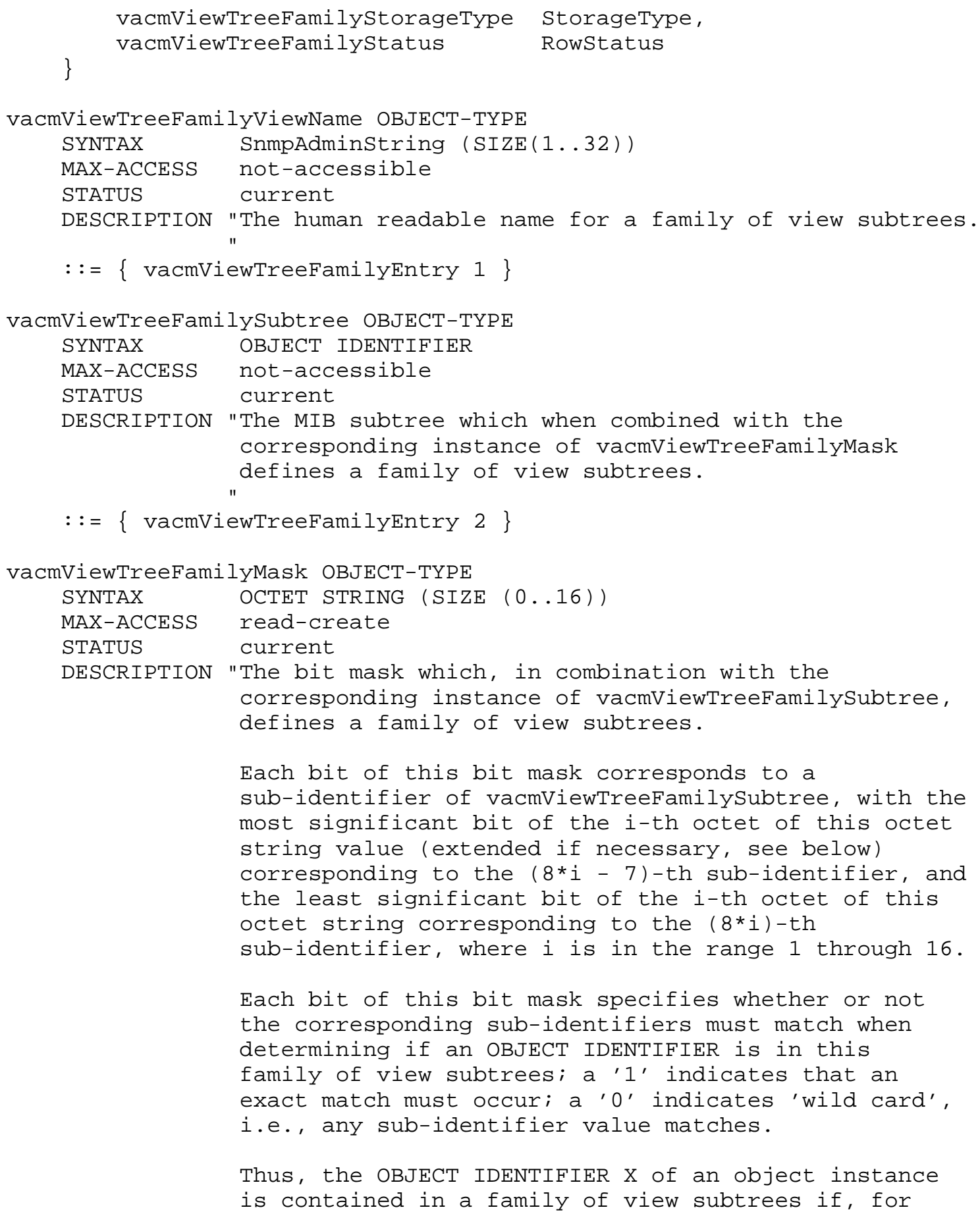




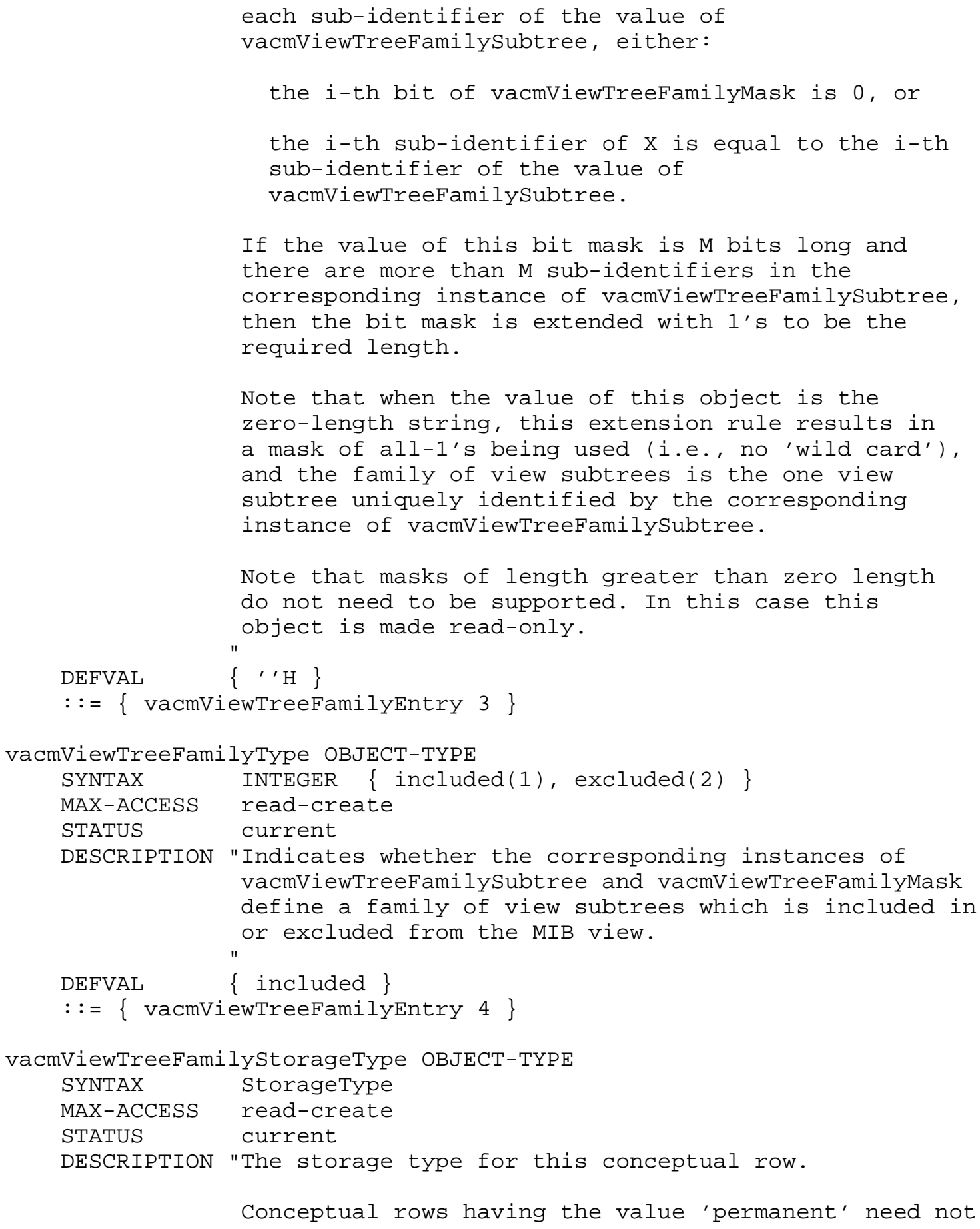




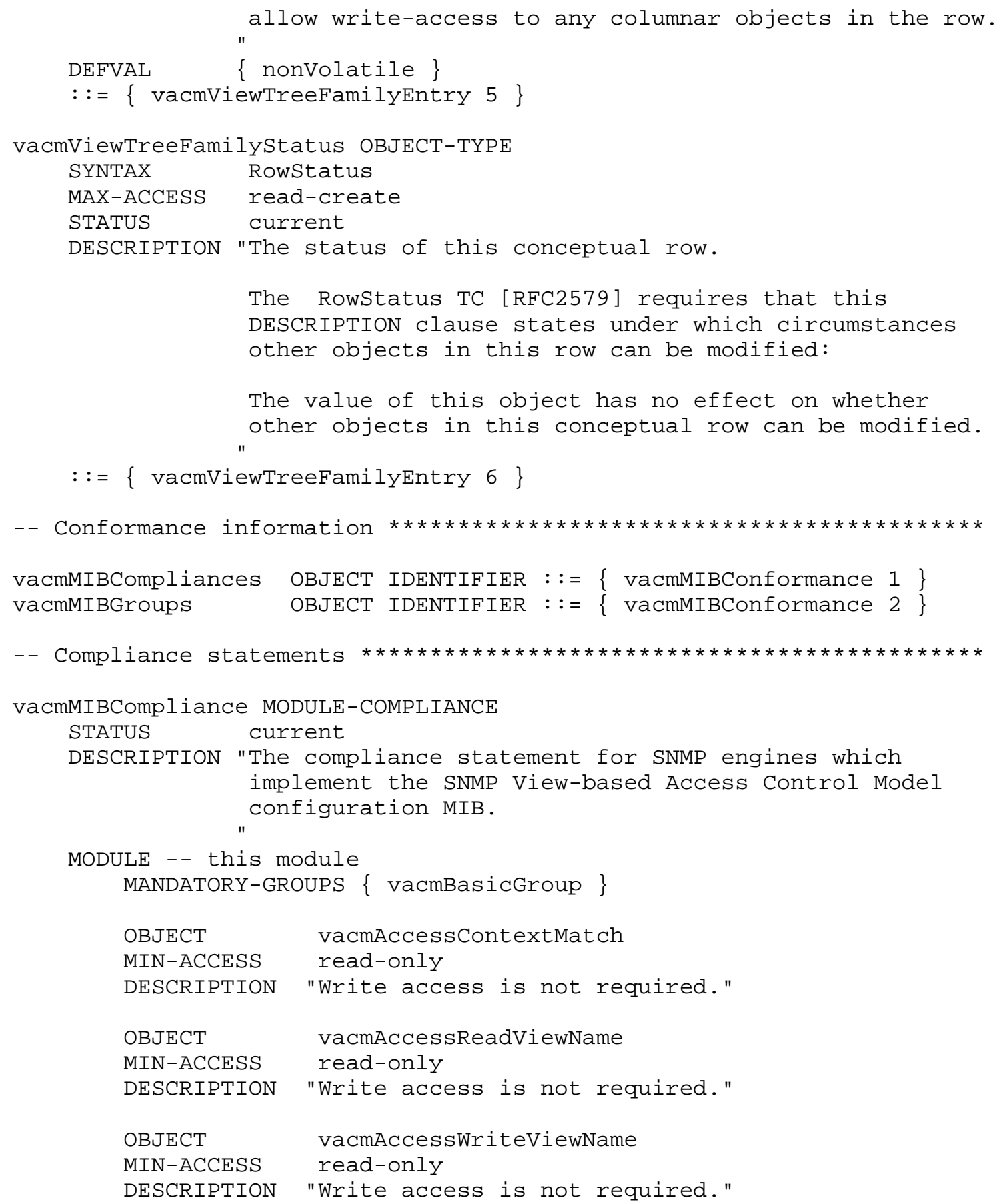




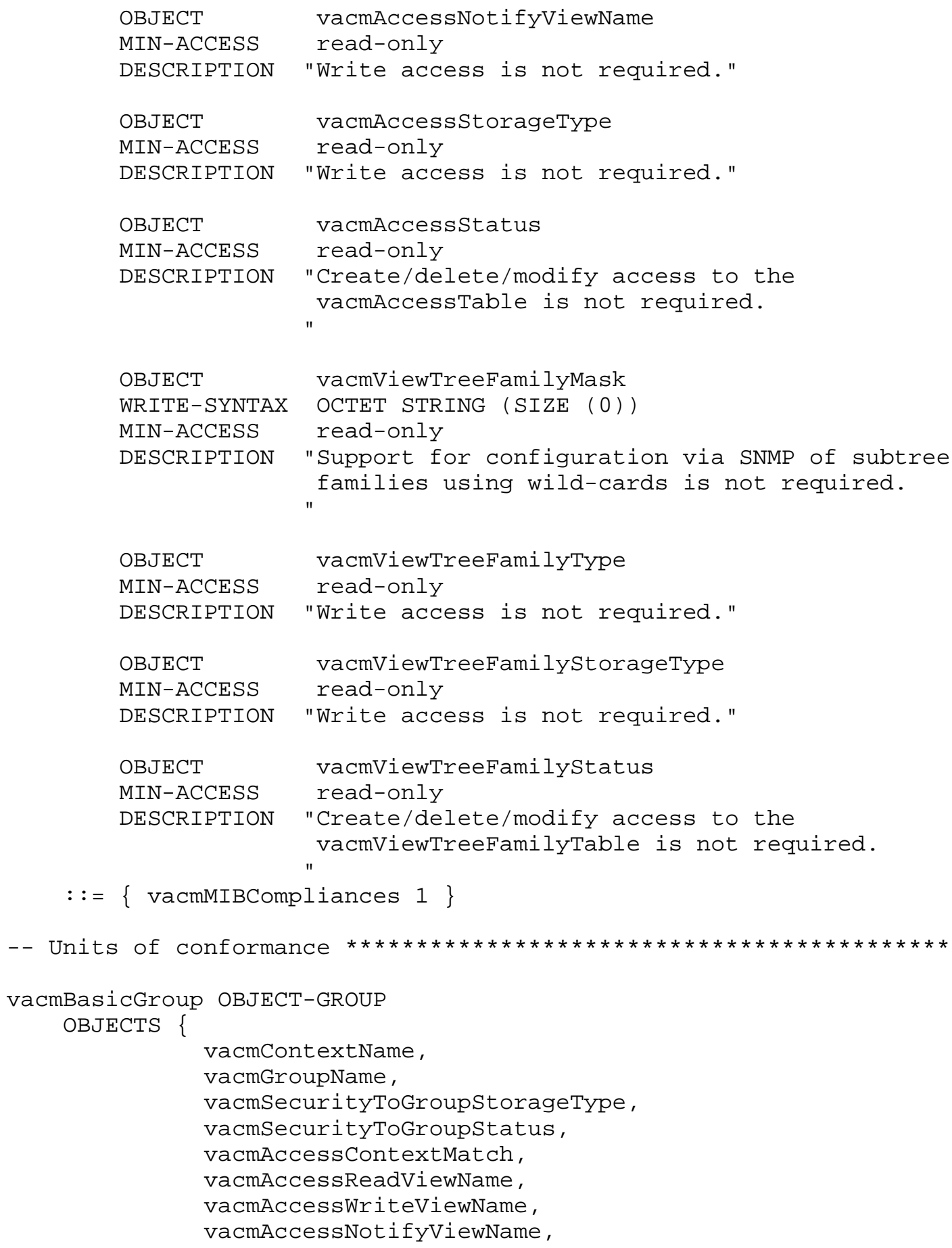




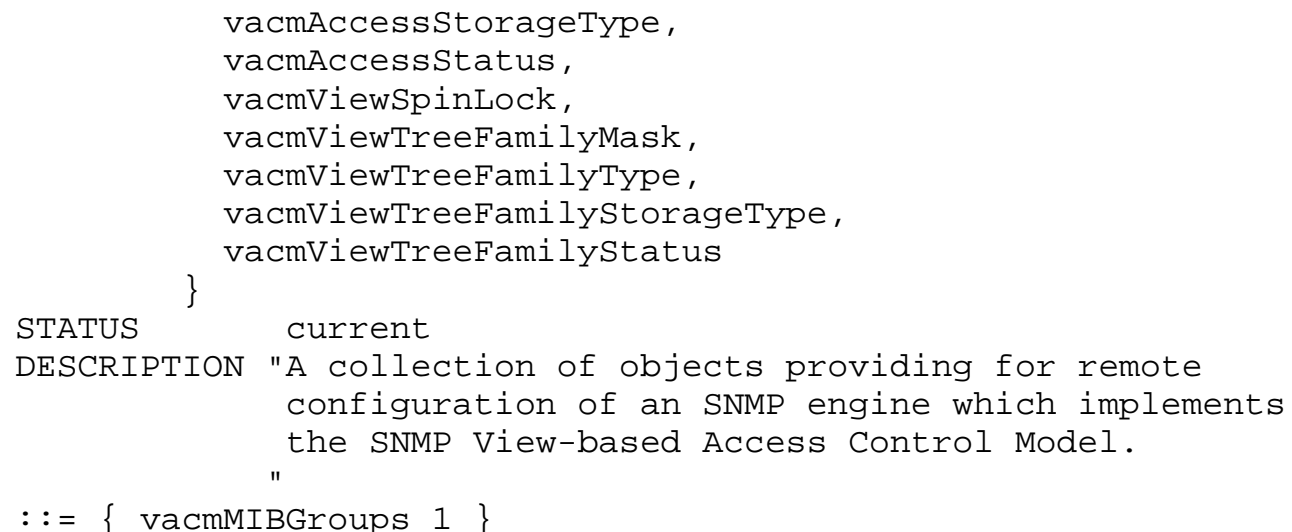

END

5. Intellectual Property

The IETF takes no position regarding the validity or scope of any intellectual property or other rights that might be claimed to pertain to the implementation or use of the technology described in this document or the extent to which any license under such rights might or might not be available; neither does it represent that it has made any effort to identify any such rights. Information on the IETF's procedures with respect to rights in standards-track and standards-related documentation can be found in BCP-11. Copies of claims of rights made available for publication and any assurances of licenses to be made available, or the result of an attempt made to obtain a general license or permission for the use of such proprietary rights by implementors or users of this specification can be obtained from the IETF Secretariat.

The IETF invites any interested party to bring to its attention any copyrights, patents or patent applications, or other proprietary rights which may cover technology that may be required to practice this standard. Please address the information to the IETF Executive Director. 


\section{Acknowledgements}

This document is the result of the efforts of the SNMPv3 Working Group. Some special thanks are in order to the following SNMPv3 WG members :

Harald Tveit Alvestrand (Maxware)

Dave Battle (SNMP Research, Inc.)

Alan Beard (Disney Worldwide Services)

Paul Berrevoets (SWI Systemware/Halcyon Inc.)

Martin Bjorklund (Ericsson)

Uri Blumenthal (IBM T.J. Watson Research Center)

Jeff Case (SNMP Research, Inc.)

John Curran (BBN)

Mike Daniele (Compaq Computer Corporation)

T. Max Devlin (Eltrax Systems)

John Flick (Hewlett Packard)

Rob Frye (MCI)

Wes Hardaker (U.C.Davis, Information Technology - D.C.A.S.)

David Harrington (Cabletron Systems Inc.)

Lauren Heintz (BMC Software, Inc.)

N.C. Hien (IBM T.J. Watson Research Center)

Michael Kirkham (InterWorking Labs, Inc.)

Dave Levi (SNMP Research, Inc.)

Louis A Mamakos (UUNET Technologies Inc.)

Joe Marzot (Nortel Networks)

Paul Meyer (Secure Computing Corporation)

Keith McCloghrie (Cisco systems)

Bob Moore (IBM)

Russ Mundy (TIS Labs at Network Associates)

Bob Natale (ACE*COMM Corporation)

Mike O'Dell (UUNET Technologies Inc.)

Dave Perkins (DeskTalk)

Peter Polkinghorne (Brunel University)

Randy Presuhn (BMC Software, Inc.)

David Reeder (TIS Labs at Network Associates)

David Reid (SNMP Research, Inc.)

Aleksey Romanov (Quality Quorum)

Shawn Routhier (Epilogue)

Juergen Schoenwaelder (TU Braunschweig)

Bob Stewart (Cisco Systems)

Mike Thatcher (Independent Consultant)

Bert Wijnen (IBM T.J. Watson Research Center) 
The document is based on recommendations of the IETF Security and Administrative Framework Evolution for SNMP Advisory Team. Members of that Advisory Team were:

David Harrington (Cabletron systems Inc.)

Jeff Johnson (Cisco Systems)

David Levi (SNMP Research Inc.)

John Linn (Openvision)

Russ Mundy (Trusted Information Systems) chair

Shawn Routhier (Epilogue)

Glenn Waters (Nortel)

Bert Wijnen (IBM T. J. Watson Research Center)

As recommended by the Advisory Team and the SNMPv3 Working Group Charter, the design incorporates as much as practical from previous RFCs and drafts. As a result, special thanks are due to the authors of previous designs known as SNMPv2u and SNMPv2*:

Jeff Case (SNMP Research, Inc.)

David Harrington (Cabletron Systems Inc.)

David Levi (SNMP Research, Inc.)

Keith McCloghrie (Cisco Systems)

Brian $O^{\prime}$ Keefe (Hewlett Packard)

Marshall T. Rose (Dover Beach Consulting)

Jon Saperia (BGS Systems Inc.)

Steve Waldbusser (International Network Services)

Glenn W. Waters (Bell-Northern Research Ltd.)

\section{Security Considerations}

\subsection{Recommended Practices}

This document is meant for use in the SNMP architecture. The Viewbased Access Control Model described in this document checks access rights to management information based on:

- contextName, representing a set of management information at the managed system where the Access Control module is running.

- groupName, representing a set of zero or more securityNames. The combination of a securityModel and a securityName is mapped into a group in the View-based Access Control Model.

- securitymodel under which access is requested.

- securityLevel under which access is requested.

- operation performed on the management information.

- MIB views for read, write or notify access. 
When the User-based Access Control module is called for checking access rights, it is assumed that the calling module has ensured the authentication and privacy aspects as specified by the securitylevel that is being passed.

When creating entries in or deleting entries from the vacmViewTreeFamilyTable it is important to do such in the sequence as recommended in the DESCRIPTION clause of the vacmViewTreeFamilyTable definition. Otherwise unwanted access may be granted while changing the entries in the table.

\subsection{Defining Groups}

The groupNames are used to give access to a group of zero or more securityNames. Within the View-Based Access Control Model, a groupName is considered to exist if that groupName is listed in the vacmSecurityToGroupTable.

By mapping the combination of a securityModel and securityName into a groupName, an SNMP Command Generator application can add/delete securityNames to/from a group, if proper access is allowed.

Further it is important to realize that the grouping of <securityModel, securityName> tuples in the vacmSecurityToGroupTable does not take securitylevel into account. It is therefore important that the security administrator uses the securityLevel index in the vacmAccessTable to separate noAuthNoPriv from authPriv and/or authNoPriv access.

\subsection{Conformance}

For an implementation of the View-based Access Control Model to be conformant, it MUST implement the SNMP-VIEW-BASED-ACM-MIB according to the vacmMIBCompliance. It also SHOULD implement the initial configuration, described in appendix A.

\subsection{Access to the SNMP-VIEW-BASED-ACM-MIB}

The objects in this MIB control the access to all MIB data that is accessible via the SNMP engine and they may be considered sensitive in many environments. It is important to closely control (both read and write) access to these to these MIB objects by using appropriately configured Access Control models (for example the View-based Access Control Model as specified in this document). 
8. References

[RFC2578] MCCloghrie, K., Perkins, D. and J. Schoenwaelder, "Structure of Management Information Version 2 (SMIv2)", STD 58, RFC 2578, April 1999.

[RFC2579] McCloghrie, K., Perkins, D. and J. Schoenwaelder, "Textual Conventions for SMIv2", STD 58, RFC 2579, April 1999.

[RFC2119] Bradner, S., "Key words for use in RFCs to Indicate Requirement Levels", BCP 14, RFC 2119, March 1997.

[RFC2571] Harrington, D., Presuhn, R. and B. Wijnen, "An Architecture for describing SNMP Management Frameworks", RFC 2571, April 1999.

[RFC2572] Case, J., Harrington, D., Presuhn, R. and B. Wijnen, "Message Processing and Dispatching for the Simple Network Management Protocol (SNMP)", RFC 2572, April 1999 .

[RFC2574] Blumenthal, U. and B. Wijnen, "User-based Security Model (USM) for version 3 of the Simple Network Management Protocol (SNMPv3)", RFC 2574, April 1999.

[ISO-ASN.1] Information processing systems - Open Systems Interconnection - Specification of Abstract Syntax Notation One (ASN.1), International Organization for Standardization. International Standard 8824, (December, 1987). 


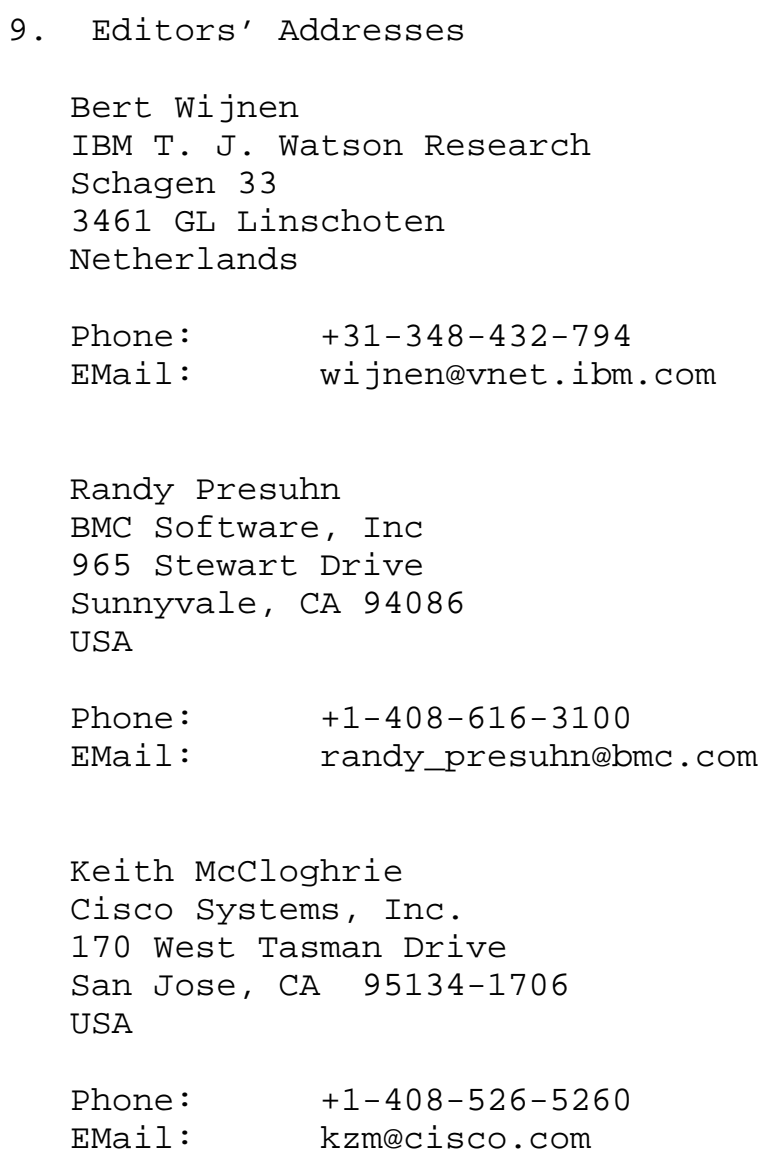


APPENDIX A - Installation

\section{A.1. Installation Parameters}

During installation, an authoritative SNMP engine which supports this View-based Access Control Model SHOULD be configured with several initial parameters. These include for the View-based Access Control Model:

1) A security configuration

The choice of security configuration determines if initial configuration is implemented and if so how. One of three possible choices is selected:

- initial-minimum-security-configuration

- initial-semi-security-configuration

- initial-no-access-configuration

In the case of a initial-no-access-configuration, there is no initial configuration, and so the following steps are irrelevant.

2) A default context

One entry in the vacmContextTable with a contextName of " " (the empty string), representing the default context. Note that this table gets created automatically if a default context exists.

vacmContextName

" "

3) An initial group

One entry in the vacmSecurityToGrouptable to allow access to group "initial".

vacmSecurityModel
vacmSecurityName
vacmGroupName
vacmSecurityToGroupstorageType
vacmSecurityToGroupstatus

vacmSecurityToGroupstatus
3 (USM)

"initial"

"initial"

anyValidstorageType

active 
4) Initial access rights

Three entries in the vacmAccessTable as follows:

- read-notify access for securityModel USM, securityLevel

"noAuthNoPriv" on behalf of securityNames that belong to the group

"initial" to the <restricted> MIB view in the default context with contextName " ".

- read-write-notify access for securityModel USM, securityLevel "authNoPriv" on behalf of securityNames that belong to the group "initial" to the <internet> MIB view in the default context with contextName " ".

- if privacy is supported, read-write-notify access for securityModel USM, securityLevel "authPriv" on behalf of securityNames that belong to the group "initial" to the <internet> MIB view in the default context with contextName " ".

That translates into the following entries in the vacmAccessTable.

- One entry to be used for unauthenticated access (noAuthNoPriv):

$\begin{array}{ll}\text { vacmGroupName } & \text { "initial" } \\ \text { vacmAccessContextPrefix } & \text { " } \\ \text { vacmAccessSecurityModel } & 3 \text { (USM) } \\ \text { vacmAccessSecurityLevel } & \text { noAuthNoPriv } \\ \text { vacmAccessContextMatch } & \text { exact } \\ \text { vacmAccessReadViewName } & \text { "restricted" } \\ \text { vacmAccessWriteViewName } & \text { " " } \\ \text { vacmAccessNotifyViewName } & \text { "restricted" } \\ \text { vacmAccessStorageType } & \text { anyValidStorageType } \\ \text { vacmAccessStatus } & \text { active }\end{array}$

- One entry to be used for authenticated access (authNopriv) with optional privacy (authPriv) :

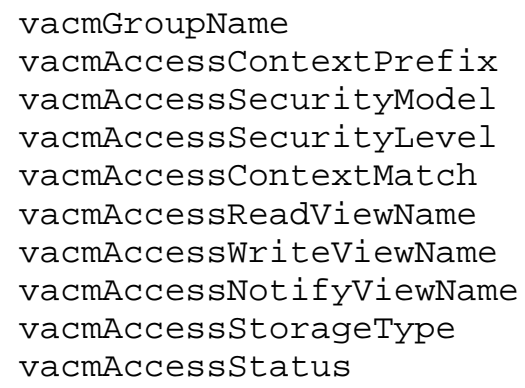

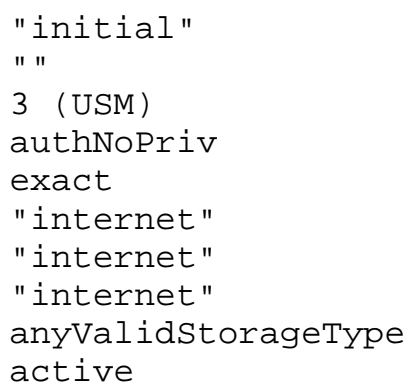


5) Two MIB views, of which the second one depends on the security configuration.

- One view, the <internet> view, for authenticated access:

- the <internet> MIB view is the following subtree:

"internet" (subtree 1.3.6.1)

- A second view, the <restricted> view, for unauthenticated access. This view is configured according to the selected security configuration:

- For the initial-no-access-configuration there is no default initial configuration, so no MIB views are pre-scribed.

- For the initial-semi-secure-configuration:

the <restricted> MIB view is the union of these subtrees:

(a) "system" (subtree 1.3.6.1.2.1.1) [RFC1907]
[

(b) "snmp" (subtree 1.3.6.1.2.1.11) [RFC1907]

(c) "snmpEngine" (subtree 1.3.6.1.6.3.10.2.1) [RFC2571]

(d) "snmpMPDStats" (subtree 1.3.6.1.6.3.11.2.1) [RFC2572]

(e) "usmStats" (subtree 1.3.6.1.6.3.15.1.1) [RFC2574]

- For the initial-minimum-secure-configuration:

the <restricted> MIB view is the following subtree. "internet" (subtree 1.3.6.1)

This translates into the following "internet" entry in the vacmViewTreeFamilyTable:

vacmViewTreeFamilyViewName vacmViewTreeFamilySubtree vacmViewTreeFamilyMask vacmViewTreeFamilyType vacmViewTreeFamilystorageType vacmViewTreeFamilystatus

minimum-secure
"internet"
1.3 .6 .1
"
1 (included)
anyValidStorageType
active

semi-secure --------------

"internet"

1.3 .6 .1

" "

1 (included)

anyValidStorageType active 
In addition it translates into the following "restricted" entries in the vacmViewTreeFamilyTable:

\begin{tabular}{|c|c|c|}
\hline & minimum-secure & semi-secure \\
\hline vacmViewTreeFamilyViewName & "restricted" & "restricted" \\
\hline $\begin{array}{l}\text { vacmViewTreeFamilysubtree } \\
\text { vacmViewTreeFamilyMask }\end{array}$ & $\begin{array}{l}1.3 \cdot 6 \cdot 1 \\
" \mathrm{~m}\end{array}$ & $1.3 \cdot 6 \cdot 1 \cdot 2 \cdot 1 \cdot 1$ \\
\hline vacmViewTreeFamilyType & 1 (included) & 1 (included) \\
\hline $\begin{array}{l}\text { vacmViewTreeFamilystorage Type } \\
\text { vacmViewTreeFamilystatus }\end{array}$ & $\begin{array}{l}\text { anyValidStorageType } \\
\text { active }\end{array}$ & $\begin{array}{l}\text { anyValidStorageType } \\
\text { active }\end{array}$ \\
\hline vacmViewTreeFamilyViewName & & "restricted" \\
\hline vacmViewTreeFamilysubtree & & $1 \cdot 3 \cdot 6 \cdot 1 \cdot 2 \cdot 1 \cdot 11$ \\
\hline vacmViewTreeFamilyMask & & " " \\
\hline vacmViewTreeFamilyType & & 1 (included) \\
\hline vacmViewTreeFamilystorageType & & anyValidStorageType \\
\hline vacmViewTreeFamilystatus & & active \\
\hline vacmViewTreeFamilyViewName & & "restricted" \\
\hline $\begin{array}{l}\text { vacmViewTreeFamilysubtree } \\
\text { vacmViewTreeFamilyMask }\end{array}$ & & $1.3 \cdot 6 \cdot 1 \cdot 6 \cdot 3 \cdot 10 \cdot 2 \cdot 1$ \\
\hline vacmViewTreeFamilyType & & 1 (included) \\
\hline vacmViewTreeFamilystorageType & & anyValidStorageType \\
\hline vacmViewTreeFamilystatus & & active \\
\hline vacmViewTreeFamilyViewName & & "restricted" \\
\hline vacmViewTreeFamilysubtree & & $1.3 \cdot 6 \cdot 1 \cdot 6 \cdot 3 \cdot 11 \cdot 2 \cdot 1$ \\
\hline vacmViewTreeFamilyType & & 1 (included) \\
\hline vacmViewTreeFamilystorageType & & anyValidstorageType \\
\hline vacmViewTreeFamilystatus & & active \\
\hline vacmViewTreeFamilyViewName & & "restricted" \\
\hline vacmViewTreeFamilysubtree & & $1 \cdot 3 \cdot 6 \cdot 1 \cdot 6 \cdot 3 \cdot 15 \cdot 1 \cdot 1$ \\
\hline vacmViewTreeFamilyMask & & " " \\
\hline vacmViewTreeFamilyType & & 1 (included) \\
\hline vacmViewTreeFamilystorageType & & anyValidStorageType \\
\hline vacmViewTreeFamilystatus & & active \\
\hline
\end{tabular}


B. Change Log

Changes made since RFC2275:

- Added text to vacmSecurityTogroupstatus DESCRIPTION clause to clarify under which conditions an entry in the vacmSecurityToGroupTable can be made active.

- Added REVISION clauses to MODULE-IDENTITY

- Clarified text in vacmAccessTable DESCRIPTION clause.

- Added a DEFVAL clause to vacmAccessContextMatch object.

- Added missing columns in Appendix A and re-arranged for clarity.

- Fixed oids in appendix A.

- Use the PDU Class terminology instead of RFC1905 PDU types.

- Added section 7.4 about access control to the MIB.

- Fixed references to new/revised documents

- Fix Editor contact information.

- fixed spelling errors

- removed one vacmAccesEntry from sample in appendix A.

- made some more clarifications.

- updated acknowledgement section. 
C. Full Copyright statement

Copyright (C) The Internet Society (1999). All Rights Reserved.

This document and translations of it may be copied and furnished to others, and derivative works that comment on or otherwise explain it or assist in its implementation may be prepared, copied, published and distributed, in whole or in part, without restriction of any kind, provided that the above copyright notice and this paragraph are included on all such copies and derivative works. However, this document itself may not be modified in any way, such as by removing the copyright notice or references to the Internet society or other Internet organizations, except as needed for the purpose of developing Internet standards in which case the procedures for copyrights defined in the Internet Standards process must be followed, or as required to translate it into languages other than English.

The limited permissions granted above are perpetual and will not be revoked by the Internet society or its successors or assigns.

This document and the information contained herein is provided on an "AS IS" basis and THE INTERNET SOCIETY AND THE INTERNET ENGINEERING TASK FORCE DISCLAIMS ALL WARRANTIES, EXPRESS OR IMPLIED, INCLUDING BUT NOT LIMITED TO ANY WARRANTY THAT THE USE OF THE INFORMATION HEREIN WILL NOT INFRINGE ANY RIGHTS OR ANY IMPLIED WARRANTIES OF MERCHANTABILITY OR FITNESS FOR A PARTICULAR PURPOSE.

Acknowledgement

Funding for the RFC Editor function is currently provided by the Internet society. 